\title{
In vitro activity of colistin in combination with various antimicrobials against Acinetobacter baumannii species, a report from South Iran
}

This article was published in the following Dove Medical Press journal: Infection and Drug Resistance

\author{
Raziyeh Kheshti' \\ Bahman Pourabbas ${ }^{2}$ \\ Maryam Mosayebi ${ }^{3}$ \\ Afsaneh Vazin' \\ 'Department of Clinical Pharmacy, \\ School of Pharmacy, Shiraz University \\ of Medical Sciences, Shiraz, \\ Iran; ${ }^{2}$ Professor Alborzi Clinical \\ Microbiology Research Center, Shiraz \\ University of Medical Sciences, Shiraz, \\ Iran; ${ }^{3}$ Student Research Committee, \\ Shiraz University of Medical Sciences, \\ Shiraz, Iran
}

Correspondence: Afsaneh Vazin

Department of Clinical Pharmacy,

School of Pharmacy, Shiraz University of

Medical Sciences, P.O. Box: 64685, Shiraz

7| 468, Iran

Tel +987132424128

Fax +98 7I3 2424126

Email vazeena@sums.ac.ir

Bahman Pourabbas

Professor Alborzi Clinical Microbiology

Research Center, Shiraz University of

Medical Sciences, Shiraz, Iran

Tel +987136474296

Fax +98 7136474303

Email bpourabbas@yahoo.com
Introduction: Acinetobacter baumannii is a gram-negative,opportunistic pathogen responsible for resistant nosocomial infections especially in the intensive care units (ICUS). One reason for the failure in the treatment of $A$. baumannii is its ability of develop resistance against several antimicrobials. combination of different antimicrobials can be used to overcome such a resistance. This study was done to evaluate the in vitro synergistic activity of colistin in combination with six different antimicrobials, including ciprofloxacin, levofloxacin, imipenem, meropenem, ampicillin-sulbactam, and rifampin against $A$. baumannii species isolated from blood culture of patients admitted to ICUs of Nemazee hospital, Shiraz, Iran.

Method: After performing biochemical identification assays on 20 isolates of A. baumannii, minimum inhibitory concentrations were determined by E- test method and antibiotic interactions were assessed using broth microdilution checkerboard method.

Results: Combinations of colistin with all six studied antimicrobials had some synergistic effect. Conclusion: clinical studies are required to clarify the therapeutic potential of these antimicrobial combinations

Keywords: Acinetobacter baumannii, colistin, synergism, anti-infective agents, intensive care unit, drug resistance

\section{Introduction}

Acinetobacter species are a group of aerobic, oxidase-negative, nonlactose fermenting, Gram-negative coccobacilli able to cause infections in community and hospital settings. ${ }^{1,2}$ Although more than 30 Acinetobacter species have been identified, most clinical infections are caused by organisms within the $A$. baumannii complex (ABC). This complex includes four species: A. baumannii, A. nosocomialis, $A$. pittii, and A. calcoaceticus, the last of which is an environmental organism of negligible clinical significance. A. baumannii is the most common genomic species in most regions of the world and is associated with higher mortality and antimicrobial resistance compared with others. ${ }^{2,3}$ It is a major cause of nosocomial infections, especially in intensive care units (ICU). ${ }^{4,5}$ However, the standard biochemical identification techniques used in clinical microbiology laboratories do not distinguish $A$. baumannii from other Acinetobacter species. ${ }^{2}$

A. baumannii is characterized by its great intrinsic and acquired resistance to many antibiotics. ${ }^{3,6}$ Infectious Diseases Society of America classified A. baumannii among the six most problematic multidrug-resistant (MDR) pathogens in hospitals worldwide. ${ }^{7}$

Increasing resistance of Acinetobacter to antibiotics stems from various mechanisms such as developing extended-spectrum $\beta$-lactamases, carbapenemases and aminoglycoside-modifying enzymes, overexpression of active efflux pumps or changes in 
outer-membrane proteins, and penicillin-binding proteins. ${ }^{1,8,9}$ Currently, many A. baumannii isolates are resistant to all aminoglycosides, cephalosporins, and fluoroquinolones. Resistance to the carbapenems and combinations of a $\beta$-lactam/ $\beta$-lactamase inhibitor, such as ampicillin-sulbactam, are also increasing. ${ }^{8,9}$ The advent of MDR bacteria and drop in the development of new antimicrobial agents have compelled the reintroduction of previously abandoned antibiotics active against resistant pathogens. Colistin, previously abandoned in clinical use because of a high rate of nephrotoxicity and neurotoxicity is currently a last-line therapy against Gram-negative bacteria such as $A$. baumannii. ${ }^{8,10}$

In a recent study of 93 isolates of $A B C$ in Nemazee Hospital (Shiraz, Iran), most of them (98.9\%) were MDR isolates, resistant to three or more of the tested antibiotics. Among them, the least resistance was observed to be to colistin (only $1.1 \%$ of the isolates). ${ }^{11}$ Although there is great controversy over combination therapy or monotherapy of resistant $A$. baumannii infections, colistin-based combination therapy is a commonly used strategy in clinical practice. ${ }^{12,13}$ Furthermore, combination therapy may prevent the emergence of resistance under therapy. ${ }^{4}$ So, we aimed to compare the in vitro activity of colistin and the combination of colistin with ciprofloxacin, levofloxacin, imipenem, meropenem, ampicillin-sulbactam, and rifampin against $A$. baumannii species isolated from blood cultures of patients admitted to the ICUs of Nemazee Hospital, a major referral hospital in the south of Iran, affiliated with Shiraz University of Medical Sciences. Previous studies have shown the in vitro synergistic effect of colistin with various antibiotics, such as imipenem, rifampin, fosfomycin, and tigecycline. ${ }^{14-16}$ In this descriptive, cross-sectional study, we examined colistin monotherapy and also the combination of colistin with six previously mentioned antimicrobial agents, one of which (levofloxacin) has not previously been studied in combination with colistin for A. baumannii infections.

\section{Methods and materials}

\section{Bacterial isolates}

This study was done on 20 isolates of lyophilized $A$. baumannii available in Doctor Alborzi Microbiology Research Laboratory in Nemazee Hospital. All of these isolates were separated from blood culture of patients hospitalized in the ICUs of Nemazee Hospital. Samples were collected as part of diagnostic tests performed as a standard of care in the hospital. The data became anonymous before the study, and therefore patient consent form was not required.

\section{Stock preparation}

Two stocks were prepared from each sample by the following method. Each of those 20 samples were separately cultured on Blood Agar medium (Merck KGaA, Darmstadt, Germany) and placed in the incubator (Memmert $\mathrm{GmbH}$, Schwabach, Germany) with temperature of $37^{\circ} \mathrm{C}$ for 24 hours. After that, to avoid the possible contamination, each sample was cultured on MacConkey Agar (Merck) and placed in the incubator for 24 hours. It is notable that MacConkey Agar is a special medium that is suitable just for growth of Gram-negative microorganisms. Then samples were cultured on Blood Agar plates again. One colony of $A$. baumannii was transferred from each plate (by using a sterile swab) to sterile microtubes (ExtraGene, Taichung City, Taiwan) containing Tripticase Soy Broth culture medium plus 15\% glycerol (Merck).

\section{Biochemical identification tests}

To identify the isolates of Acinetobacter, Gram staining, oxidase test, and PCR were done.

Identification of Acinetobacter isolates at the species level was made by the PCR amplification of $g y r-B$ gene. We used the method previously described by Higgins et al for multiplex PCR based on species-specific gyrB primers for confirmation of the identification of A. baumannii species. ${ }^{17}$

\section{MIC determination}

Minimum inhibitory concentration (MIC) is the lowest concentration of an antibiotic able to inhibit visible microorganism growth after 24 hours of incubation. We used E-test method to determine MIC. For this purpose, all A. baumannii samples were cultured on Blood Agar medium and incubated for 24 hours at $37^{\circ} \mathrm{C}$. Then two or three colonies of the cultured samples were transferred to Mueller-Hinton Broth (Merck) and placed in the incubator to get $1.5 \times 10^{8}$ colony-forming units $(\mathrm{CFU}) /$ $\mathrm{mL}$. In the next step, each sample was cultured on three Muller-Hinton Broth plates. Two E-test strips containing different antibiotics were placed on each plate. After 24 hours of incubation, MIC number of each antibiotic was read on the strip.

\section{Synergy testing of colistin combinations with the broth microdilution checkerboard method}

Synergistic effects were determined by the broth microdilution checkerboard method ${ }^{18}$ performed in 96 -well microtiter 
plates (SPL, Kyungki, South Korea) containing colistin and one of six other antibiotics (ciprofloxacin, levofloxacin, imipenem, meropenem, ampicillin-sulbactam, and rifampin). All antibiotics were obtained from Abcam, Cambridge, UK. Each antibiotic was diluted to concentrations ranging from $1 / 4$ or $1 / 5$ of MIC to four or five times more than the previously determined MIC (as $8 \times \mathrm{MIC}, 4 \times \mathrm{MIC}, 2 \times \mathrm{MIC}$, $1 \times \mathrm{MIC}, 0.5 \times \mathrm{MIC}, 0.25 \times \mathrm{MIC}, 0.125 \times \mathrm{MIC}$, and $0 \mathrm{mg} /$ $\mathrm{mL})$. All of these steps were done under sterile condition using Class II Microbiological Safety Cabinets (Telstar, Terrassa, Spain). After 24-hour incubation at $37^{\circ} \mathrm{C}$, MICs were determined. The initial inoculum was $\sim 5 \times 10^{5} \mathrm{CFU} /$ $\mathrm{mL}$. For each of the six combinations, the fractional inhibitory concentration index (FICI) was calculated according to the following equation:

FICI $=\frac{\text { MIC of drug A in combination }}{\text { MIC of drug A alone }}+\frac{\text { MIC of drug B in combination }}{\text { MIC of drug B alone }}$

The FICI results for each combination were interpreted as follows: $\mathrm{FICI} \leq 0.5$, synergism; $0.5<\mathrm{FICI}<1$, partial synergism; $1 \leq \mathrm{FICI}<2$, indifference; and FICI $\geq 2$, antagonism. ${ }^{19}$ Escherichia coli ATCC 25922 and Pseudomonas aeruginosa ATCC 27853 were used as standard control strains for the assays.

\section{Results}

\section{Bacterial isolates and stock preparation}

Twenty blood-culture-derived isolates of $A$. baumannii available in Doctor Alborzi Microbiology Research Lab were selected. Two stocks were prepared from each sample by the previously explained method.

\section{Biochemical identification tests}

Oxidase test was done on all 20 isolates of $A$. baumannii. It was confirmed that all isolates were oxidase negative. $A$. baumannii isolates, which are Gram-negative, appeared pink in Gram stain.

The multiplex PCR based on difference in gyr-B gene was done to identify species of Acinetobacter isolates. All isolates of $A$. baumannii showed two bands of 240 and 640 $\mathrm{bp}$, which are specific to this species.

\section{MIC determination}

E-test assay was done on each sample, and MIC of $A$. baumannii to all seven antibiotics was determined. Results are shown in Table 1.

Table I The minimum inhibitory concentration (MIC) values $(\mathrm{mcg} / \mathrm{mL})$ obtained using E-test method of antimicrobial agents against 20 Acinetobacter baumannii samples isolated from blood cultures of patients admitted to intensive care units of Nemazee Hospital

\begin{tabular}{|c|c|c|c|c|c|c|c|}
\hline \multirow[t]{2}{*}{ Sample } & \multicolumn{7}{|c|}{ MIC } \\
\hline & Rifampin & Ciprofloxacin & Levofloxacin & Imipenem & Meropenem & $\begin{array}{l}\text { Ampicillin- } \\
\text { sulbactam }\end{array}$ & Colistin \\
\hline I & 1 & 0.2 & 0.5 & 2 & 0.009 & 0.009 & 0.25 \\
\hline 2 & I & 0.256 & 16 & 2 & 0.18 & 6 & 0.5 \\
\hline 3 & I & 0.4 & 0.05 & 8 & 0.025 & 2 & 0.125 \\
\hline 4 & 0.5 & 0.125 & 0.06 & 1 & 0.03 & 3 & 0.25 \\
\hline 5 & I & 0.2 & 0.05 & 8 & 0.015 & 1 & 0.25 \\
\hline 6 & 4 & 64 & 8 & 8 & 0.18 & 6 & 0.125 \\
\hline 7 & 4 & 128 & 32 & 8 & 0.25 & 4 & 0.25 \\
\hline 8 & I & 0.25 & 1 & 0.625 & 0.03 & I & 0.18 \\
\hline 9 & I & 0.5 & 0.03 & 2 & 0.015 & 0.5 & 1 \\
\hline 10 & I & 0.064 & 0.128 & 8 & 0.19 & 1.5 & 0.06 \\
\hline II & I & 0.188 & 0.094 & 4 & 0.19 & 0.75 & 0.25 \\
\hline 12 & 16 & I & 0.5 & 8 & 0.025 & 6 & 0.375 \\
\hline 13 & 2 & 128 & 6 & 0.15 & 0.09 & 2 & 1 \\
\hline 14 & 0.5 & 0.095 & 0.76 & 8 & 0.015 & I & 0.18 \\
\hline 15 & 1 & 64 & 8 & 8 & 8 & 12 & 0.25 \\
\hline 16 & 2 & 32 & 64 & 24 & 8 & 64 & 0.19 \\
\hline 17 & 1.5 & 64 & 32 & 48 & 32 & 64 & 0.375 \\
\hline 18 & 8 & 64 & 8 & 32 & 32 & 16 & 0.5 \\
\hline 19 & 0.5 & 128 & 8 & 16 & 8 & 24 & 0.093 \\
\hline 20 & $>128$ & 32 & 2 & 32 & 32 & 64 & 0.06 \\
\hline
\end{tabular}




\section{Checkerboard synergy test}

The synergistic effect of each of six antibiotics in combination with colistin was determined by the broth microdilution checkerboard method. Results of the checkerboard synergy test of colistin combination with other six antibiotics against all the 20 isolates of $A$. baumannii are shown in Table 2. Comparison of synergy test results of all six combinations are summarized in Table 3.

\section{Discussion}

A. baumannii is a common pathogen in the ICU and is associated with high mortality and antimicrobial resistance..$^{2-4}$ Many
A. baumannii isolates are now resistant to various antimicrobials such as aminoglycosides, cephalosporins, fluoroquinolones, carbapenems, and $\beta$-lactam $/ \beta$-lactamase inhibitor combinations. Colistin is among limited last-line options active against MDR Gram-negative pathogens such as $A$. baumannii. ${ }^{8,9}$ As the rate of MDR $A$. baumannii isolates were seen to be high ( $98.9 \%$ of the isolated $\mathrm{ABCs})$ in our center ${ }^{11}$ and colistin-based combination therapy is commonly used in clinical practice for this isolates, ${ }^{12,13}$ we aimed to evaluate the in vitro synergistic effects of antimicrobial combinations against $A$. baumannii isolated from blood cultures of patients admitted to the ICUs of Nemazee Hospital, a major referral

Table 2 Checkerboard synergy study results for the combinations of colistin with six different antibiotics (rifampin, ciprofloxacin, levofloxacin, imipenem, meropenem, ampicillin-sulbactam) tested against 20 samples of Acinetobacter Baumannii strains isolated from blood cultures of patients admitted to intensive care units of Nemazee Hospital

\begin{tabular}{|c|c|c|c|c|c|c|c|c|c|c|c|c|}
\hline \multirow[t]{3}{*}{ Sample } & \multicolumn{12}{|c|}{ Combination of antibiotics with colistin } \\
\hline & \multicolumn{2}{|c|}{ Rifampin } & \multicolumn{2}{|c|}{ Ciprofloxacin } & \multicolumn{2}{|c|}{ Levofloxacin } & \multicolumn{2}{|c|}{ Imipenem } & \multicolumn{2}{|c|}{ Meropenem } & \multicolumn{2}{|c|}{$\begin{array}{l}\text { Ampicillin- } \\
\text { sulbactam }\end{array}$} \\
\hline & $\mathbf{F I C I}$ & Result & $\mathbf{F I C l}$ & Result & $\mathbf{F I C I}$ & Result & $\mathbf{F I C I}$ & Result & $\mathbf{F I C I}$ & Result & $\mathbf{F I C I}$ & Result \\
\hline 1 & 0.75 & part & 0.62 & part & 0.37 & syn & 2.5 & anta & I & ind & I & ind \\
\hline 2 & 0.75 & part & I & ind & I & ind & 0.5 & syn & I & ind & I & ind \\
\hline 3 & 0.49 & syn & 0.07 & syn & 2.5 & anta & 1 & ind & 2.5 & anta & 1.5 & ind \\
\hline 4 & 0.62 & part & 0.56 & part & 2 & anta & 1 & ind & 2.5 & anta & I & ind \\
\hline 5 & 0.37 & syn & 0.75 & part & 2 & anta & 2.007 & anta & 0.75 & part & 1.5 & ind \\
\hline 6 & 0.6 & part & 0.56 & part & 0.74 & part & 2.5 & anta & 2.5 & anta & I & ind \\
\hline 7 & 0.5 & syn & 4.25 & anta & 2 & anta & 2.5 & anta & I & ind & 0.75 & part \\
\hline 8 & 0.37 & syn & 1.25 & ind & I & ind & 0.75 & part & 2.5 & anta & 0.375 & syn \\
\hline 9 & 0.5 & syn & 0.5 & syn & 2 & anta & 3 & anta & I & ind & 0.31 & syn \\
\hline 10 & 0.37 & syn & 2 & anta & 0.75 & part & I & ind & I & ind & 2.5 & anta \\
\hline 11 & 0.31 & syn & 2.06 & anta & 1 & ind & 1 & ind & 3 & anta & 2.5 & anta \\
\hline 12 & 0.25 & part & 1 & ind & 2 & anta & 2.06 & anta & 2.25 & anta & 1 & ind \\
\hline 13 & 0.625 & part & 2.125 & anta & 4 & anta & 2 & anta & 1 & ind & 0.5 & syn \\
\hline 14 & 0.5 & syn & 0.5 & syn & 2 & anta & 2.5 & anta & 0.75 & part & 1.25 & ind \\
\hline 15 & 0.37 & syn & 1 & ind & 0.62 & part & 0.37 & syn & 0.62 & part & 1.5 & ind \\
\hline 16 & 0.75 & part & I & ind & 2 & anta & 0.5 & syn & 0.55 & part & 1 & ind \\
\hline 17 & 0.3 & syn & 3 & anta & 0.55 & part & 0.5 & syn & 0.55 & part & 0.75 & part \\
\hline 18 & 1 & ind & 0.75 & part & 2 & anta & 0.75 & part & 0.37 & syn & 1 & ind \\
\hline 19 & 0.75 & part & 1 & ind & 4.5 & anta & 1.5 & ind & I & ind & 1 & ind \\
\hline 20 & 0.24 & syn & 2.58 & anta & 1 & ind & 0.625 & part & 0.37 & syn & 2.3 & anta \\
\hline
\end{tabular}

Abbreviations: anta, antagonist; $\mathrm{FICl}$, fractional inhibitory concentration index; ind, indifference; part, partial synergy; syn, synergy.

Table 3 Results of the checkerboard synergy test of combination of colistin with six different antibiotics against 20 strains of Acinetobacter baumannii isolated from blood cultures of patients admitted to intensive care units of Nemazee Hospital

\begin{tabular}{|l|l|l|l|l|}
\hline & Result & \multicolumn{2}{l|}{} \\
\hline Combination & Synergy & Partial synergy & Indifference & Antagonist \\
\hline Colistin + rifampin & $55 \%$ & $40 \%$ & $5 \%$ & $0 \%$ \\
Colistin + imipenem & $20 \%$ & $15 \%$ & $25 \%$ & $40 \%$ \\
Colistin + ciprofloxacin & $15 \%$ & $25 \%$ & $30 \%$ & $30 \%$ \\
Colistin + ampicillin-sulbactam & $15 \%$ & $10 \%$ & $60 \%$ & $15 \%$ \\
Colistin + meropenem & $10 \%$ & $25 \%$ & $35 \%$ & $30 \%$ \\
Colistin + levofloxacin & $5 \%$ & $20 \%$ & $65 \%$ & $10 \%$ \\
\hline
\end{tabular}


hospital in the south of Iran. So, combinations of colistin with six commonly used antimicrobial agents (ciprofloxacin, levofloxacin, imipenem, meropenem, ampicillin-sulbactam, and rifampin) were examined by checkerboard assay to find the combination with the highest synergistic effect against this pathogen.

Among the six studied combinations, colistin-rifampin showed the highest synergy. In 11 (55\%) of the $20 \mathrm{~A}$. baumannii isolates, combination of colistin with rifampin showed synergistic effect (FICI $\leq 0.5)$, and in $40 \%$ of them, partial synergy $(0.5 \leq \mathrm{FICI} \leq 1)$ was recorded. At the opposite end of the spectrum, colistin-levofloxacin combination showed the lowest synergistic effect. Only in 5\% of the isolates, synergy was seen with this combination. In $10 \%$ of them, antagonistic effect (FICI $\geq 2$ ) was seen, and in $65 \%$, effect of colistin and levofloxacin was not different either alone or in combination with each other (indifference: $1 \leq \mathrm{FICI} \leq 2)$. In addition, colistin-imipenem combination exhibited the highest (40\%) antagonistic effect. In a previous study on eleven isolates of $A$. baumannii, Hogg et al reported that the colistin-rifampin combination was synergic against eleven isolates and indifferent against two; antagonism was not detected in that study. ${ }^{20}$ Although only two isolates were categorized as susceptible to rifampin, this agent showed synergy with colistin against eleven isolates. It has previously been confirmed that rifampin combined with other antimicrobials (such as colistin, imipenem, ceftazidim, and ofloxacin) may be synergic against some MDR Gram-negative bacteria. ${ }^{21,22}$

Recently a systematic review and meta-analysis (on 70 published articles and 31 conference proceedings) reported the in vitro synergistic activity of polymyxins combined with eleven other antibiotic classes against A. baumannii. ${ }^{23}$ It was concluded that carbapenems and rifampin combination could efficiently decrease the development of colistin resistance and displayed a $>50 \%$ synergy rate against colistin-resistant strains. Pooled synergy rates of polymyxins-carbapenems and polymyxins-rifampin combinations, checked by checkerboard method, were $39.6 \%$ and $60.3 \%$, respectively. The synergy rate of rifampincolistin combination in our study was $55 \%$, which is near the result of this systematic review (60.3\%). However, the synergy rate of carbapenems-colistin combination was lower in our study (colistin-imipenem: 20\%, colistinmeropenem: 10\%). Of note, the range of synergy rate for polymyxins-carbapenems combination was very wide in this review (95\% CI: 19.6\%-63.9\%). So, our result was not improbable. On the other hand, the review considered two polymyxins (colistin and polymyxin B) together and pooled their data altogether. ${ }^{23}$ This can be another reason for the difference between their results and ours.

Our findings conflict with the results of two systemic review and meta-analysis of the in vitro synergy of polymyxins and carbapenems, which indicated significant advantages of combining meropenem with colistin, rather than imipenem, for $A$. baumannii infections..$^{23,24}$

In the current study, checkerboard synergy assay was done on colistin-levofloxacin and colistin-ciprofloxacin combinations for the first time. Colistin-levofloxacin combination indicated the lowest synergistic effect $(5 \%)$ among the all six studied antimicrobial combinations. The synergy rate for colistin-ciprofloxacin was $15 \%$. We could not find any study evaluating the synergistic effect of these two combinations and comparing it with other antimicrobial combinations.

The synergy rate of colistin with ampicillin-sulbactam was $15 \%$ in our study. A broad range of synergy rates has been reported in the previous studies on this combination, from no synergy to $92 \%$ synergy rate. ${ }^{25,26}$

In general, the discrepancy between our results and other studies' findings could be due to using different methods for synergy examination. It has been previously shown that synergy rates of E-test and checkerboard microdilution assays in most antibiotic combinations are generally lower than those of time-kill assays. ${ }^{23,24}$ Another explanation could be the variance in sample size of different studies.

A number of methods have been used to detect in vitro synergistic effect of antibiotics. However, the checkerboard and time-kill assays are the most widely used techniques. The checkerboard method is a relatively easy test to perform. Several studies have compared results generated by the checkerboard and time-kill methods. While some studies have shown excellent agreement between these methods; controversy over the comparability of results generated by these techniques exists. Each method has its advocates. However there is no agreement on which one is the best and standard method to determine the synergy evaluation. ${ }^{27}$

\section{Conclusion}

In summary, based on in vitro checkerboard assay, we found that combination of colistin with ciprofloxacin, levofloxacin, imipenem, meropenem, ampicillin-sulbactam, or rifampin had synergistic activity against $A$. baumannii isolates in our center. Among them, colistin-rifampin had the highest potential synergistic effect. However, we cannot assume their efficacy in clinical practice because in vivo settings, such as drug concentrations in the site of action, load and resistance of bacteria, and host immune response in the body, cannot 
be totally simulated in vitro. Clinical studies are required to clarify the therapeutic potential of these antimicrobial combinations and to explore mechanisms responsible for its synergy.

\section{Acknowledgments}

The present article was extracted from the thesis written by Maryam Mosayebi and was financially supported by Shiraz University of Medical Sciences, grant number 92-01-057013. Supported by Professor Alborzi Clinical Microbiology Research Center, Shiraz University of Medical Sciences, Shiraz, Iran.

\section{Author contributions}

All authors contributed to data analysis, drafting or revising the article, gave final approval of the version to be published, and agree to be accountable for all aspects of the work.

\section{Disclosure}

The authors report no conflicts of interest in this work.

\section{References}

1. Doi Y, Murray GL, Peleg AY. Acinetobacter baumannii: evolution of antimicrobial resistance-treatment options. Semin Respir Crit Care Med. 2015;36(1):85-98.

2. Viehman JA, Nguyen MH, Doi Y. Treatment options for carbapenemresistant and extensively drug-resistant Acinetobacter baumannii infections. Drugs. 2014;74(12):1315-1333.

3. Lynch JP, Zhanel GG, Clark NM. Infections due to Acinetobacter baumannii in the ICU: treatment options. Semin Respir Crit Care Med. 2017;38(3):311-325.

4. Garnacho-Montero J, Dimopoulos G, Poulakou G, et al. Task force on management and prevention of Acinetobacter baumannii infections in the ICU. Intensive Care Med. 2015;41(12):2057-2075.

5. Vazin A, Karimzadeh I, Zand A, Hatami-Mazinani N, Firouzabadi D. Evaluating adherence of health-care team to standard guideline of colistin use at intensive care units of a referral hospital in Shiraz, Southwest of Iran. Adv Pharm Bull. 2017;7(3):391-397.

6. Olaitan AO, Morand S, Rolain JM. Mechanisms of polymyxin resistance: acquired and intrinsic resistance in bacteria. Front Microbiol. 2014;5:643.

7. Boucher HW, Talbot GH, Bradley JS, et al. Bad bugs, no drugs: no ESKAPE! An update from the Infectious Diseases Society of America. Clin Infect Dis. 2009;48(1):1-12.

8. Talbot GH, Bradley J, Edwards JE, et al. Bad bugs need drugs: an update on the development pipeline from the Antimicrobial Availability Task Force of the Infectious Diseases Society of America. Clin Infect Dis. 2006;42(5):657-668.

9. Wong D, Nielsen TB, Bonomo RA, Pantapalangkoor P, Luna B, Spellberg B. Clinical and pathophysiological overview of acinetobacter infections: a century of challenges. Clin Microbiol Rev. 2017;30(1):409-447.
10. Bergen PJ, Landersdorfer CB, Lee HJ, Li J, Nation RL. "Old" antibiotics for emerging multidrug-resistant bacteria. Curr Opin Infect Dis. 2012;25(6):626-633.

11. Anvarinejad M, Japoni A, Davarpanah MA, Mahmudi H, Mammina C, Vazin A. Phenotypic and molecular epidemiology of acinetobacter calcoaceticus baumannii complex strains spread at Nemazee Hospital of Shiraz, Iran. Jundishapur J Microbiol. 2015;8(6):e19180.

12. Vourli S, Frantzeskaki F, Meletiadis J, et al. Synergistic interactions between colistin and meropenem against extensively drug-resistant and pandrug-resistant Acinetobacter baumannii isolated from ICU patients. Int J Antimicrob Agents. 2015;45(6):670-671.

13. Thamlikitkul V, Tiengrim S. In vitro activity of colistin plus sulbactam against extensive-drug-resistant Acinetobacter baumannii by checkerboard method. J Med Assoc Thai. 2014;97(Suppl 3):S1-S6.

14. Nastro M, Rodríguez CH, Monge R, et al. Activity of the colistinrifampicin combination against colistin-resistant, carbapenemase-producing Gram-negative bacteria. J Chemother. 2014;26(4):211-216.

15. Percin D, Akyol S, Kalin G. In vitro synergism of combinations of colistin with selected antibiotics against colistin-resistant Acinetobacter baumannii. GMS Hyg Infect Control. 2014;9(2):Doc14.

16. Vidaillac C, Benichou L, Duval RE. In vitro synergy of colistin combinations against colistin-resistant Acinetobacter baumannii, Pseudomonas aeruginosa, and Klebsiella pneumoniae isolates. Antimicrob Agents Chemother. 2012;56(9):4856-4861.

17. Higgins PG, Lehmann M, Wisplinghoff H, Seifert H. gyrB multiplex PCR to differentiate between Acinetobacter calcoaceticus and Acinetobacter genomic species 3. J Clin Microbiol. 2010;48(12):4592-4594.

18. Schwalbe R, Steele-Moore L, Goodwin AC. Antimicrobial Susceptibility Testing Protocols. Boca Raton, FL: CRC Press; 2007.

19. Patil SV, Hajare AL, Patankar M, Krishnaprasad K. In vitro fractional inhibitory concentration (FIC) study of cefixime and azithromycin fixed dose combination (FDC) against respiratory clinical isolates. J Clin Diagn Res. 2015;9(2):DC13-DC15.

20. Hogg GM, Barr JG, Webb CH. In-vitro activity of the combination of colistin and rifampicin against multidrug-resistant strains of Acinetobacter baumannii. J Antimicrob Chemother. 1998;41(4):494-495.

21. Mohammadi M, Khayat H, Sayehmiri K, et al. Synergistic effect of colistin and rifampin against multidrug resistant Acinetobacter baumannii: a systematic review and meta-analysis. Open Microbiol J. 2017;11:63-71.

22. Grayson ML, Crowe SM, McCarthy JS. Kucers' the Use of Antibiotics Sixth Edition: A Clinical Review of Antibacterial, Antifungal and Antiviral Drugs. Ed. US, CRC Press; 2010.

23. Ni W, Shao X, di X, Cui J, Wang R, Liu Y. In vitro synergy of polymyxins with other antibiotics for Acinetobacter baumannii: a systematic review and meta-analysis. Int J Antimicrob Agents. 2015;45(1):8-18.

24. Zusman O, Avni T, Leibovici L, et al. Systematic review and metaanalysis of in vitro synergy of polymyxins and carbapenems. Antimicrob Agents Chemother. 2013;57(10):5104-5111.

25. Cikman A, Ceylan MR, Parlak M, Karahocagil MK, Berktaş M. Evaluation of colistin-ampicillin/sulbactam combination efficacy in imipenem-resistant acinetobacter baumannii strains. Mikrobiyol Bul. 2013;47(1):147-151.

26. Wei W, Yang H, Liu Y, Ye Y, Li J. In vitro synergy of colistin combinations against extensively drug-resistant Acinetobacter baumannii producing OXA-23 carbapenemase. J Chemother. 2016;28(3):159-163.

27. Jiang Z, He X, Li J. Synergy effect of meropenem-based combinations against Acinetobacter baumannii: a systematic review and meta-analysis. Infect Drug Resist. 2018;11:1083-1095. 
Infection and Drug Resistance is an international, peer-reviewed openaccess journal that focuses on the optimal treatment of infection (bacterial, fungal and viral) and the development and institution of preventive strategies to minimize the development and spread of resistance. The journal is specifically concerned with the epidemiology of antibiotic resistance and the mechanisms of resistance development and diffusion in both hospitals and the community. The manuscript management system is completely online and includes a very quick and fair peerreview system, which is all easy to use. Visit http://www.dovepress.com/ testimonials.php to read real quotes from published authors.

Submit your manuscript here: https://www.dovepress.com/infection-and-drug-resistance-journal 